\title{
Prevalence and variables associated with solitary pulmonary nodules in a routine clinic-based population: a cross-sectional study
}

\author{
N. Gómez-Sáez • I. González-Álvarez • J. Vilar • I. Hernández-Aguado • \\ M. L. Domingo • M. F. Lorente • M. Pastor-Valero • L. A. Parker • \\ N. Picazo • J. Calbo • B. Lumbreras
}

Received: 28 December 2013 / Revised: 6 May 2014 / Accepted: 15 May 2014 / Published online: 25 June 2014

(C) The Author(s) 2014. This article is published with open access at Springerlink.com

\begin{abstract}
Objective To determine the prevalence of solitary pulmonary nodules (SPNs) in chest radiology studies and patient's features associated with malignancy in a non-high-risk clinical population.

Methods Patients $\geq 35$ years were referred for thoracic imaging in two hospitals (2010-2011). Eight radiologists determined the presence and characteristics of SPN. Selected variables were collected from radiological register and medical records. Observer agreement in the diagnosis of SPN was assessed.

Results 25,529 patients were included: 23,102 (90.5\%) underwent chest radiograph and 2,497 (9.5\%) a CT. The prevalence of SPN was $2.1 \%(95 \%$ CI $1.9-2.3)$ in radiographs and $17.0 \%(95 \%$ CI $15.5-18.5)$ in CT. In patients undergoing chest radiograph, detection of SPN with an irregular border was more frequent among smokers. In patients who had a CT, larger SPNs appeared to be associated with 60 years of age or over, diagnosis of a respiratory illness, or male gender. In addition, an irregular border was also more common among men.

Conclusions The prevalence of SPNs detected by both radiograph and CT was lower than that shown in screening studies.
\end{abstract}

N. Gómez-Sáez · I. Hernández-Aguado • M. Pastor-Valero ·

L. A. Parker $\cdot$ B. Lumbreras

Public Health Department, Miguel Hernández University, Alicante, Spain

I. González-Álvarez • M. F. Lorente • N. Picazo • J. Calbo

Radiodiagnostic Department, San Juan Hospital, Alicante, Spain

J. Vilar · M. L. Domingo

Radiodiagnostic Department, Peset Hospital, Valencia, Spain

I. Hernández-Aguado $\cdot$ M. Pastor-Valero • L. A. Parker •

B. Lumbreras $(\square)$

Ciber en Epidemiología y Salud Pública, Barcelona, Spain

e-mail: blumbreras@umh.es
Patient characteristics such as age, sex, respiratory disease, or smoking habit were associated with nodule characteristics that are known to be related with malignancy.

Key Points

- There is a lower SPN prevalence in the clinical population than in screening studies.

- SPN prevalence is associated with some patient characteristics: sex, age, imaging test.

- Nodule characteristics related to malignancy were associated with some patient characteristics.

Keywords Solitary pulmonary nodule $\cdot$ General population . Imaging $\cdot$ Lung $\cdot$ Malignancy

\section{Introduction}

Suspicion of lung cancer is a frequent event during the interpretation of thoracic imaging tests, particularly since the use of more sensitive tests like computed axial tomography (CT) has become widespread [1]. When faced with a suspicious image, radiologists must decide if it is necessary to carry out new diagnostic procedures either on their own initiative or by recommendation, or to recommend active surveillance when the suspicion is minimal. This topic has been, and continues to be, the object of a great deal of research $[2,3]$. Physicians evaluate the clinical probability of nodule malignancy either through their experience or with the help of different quantitative models, which are based on the scientific literature [4, 5]. The recommendations in the management of solitary pulmonary nodules (SPN) were established by the American College of Chest Physicians (ACCP) in 2007 [6], and have been recently updated [5]. 
Most of these predictive tools, based on patient and nodule characteristics, come from high-risk populations undergoing screening [7], which differ quite significantly from the unselected population undergoing imaging in a routine setting (i.e., people with respiratory symptoms who seek medical care, patients with other complaints, or asymptomatic patients with incidental nodules). Screening trials use very strict inclusion criteria $[8,9]$ such as the inclusion of individuals aged between $50-75$ years with at least a 30 pack-year tobacco habit. Applying the findings observed in these highly selected populations to guide clinical decisions about nodules observed in a routine clinical setting could, therefore, be flawed. The few studies that are carried out in clinic-based populations have limitations that hamper the application of their findings to a wider setting (do not describe the selection process of the patients included in the study [10-13], have small sample size $[14,15]$, are retrospective [4], or include a very specific, and thus, non-representative population [6]). Thus, there is currently insufficient evidence to develop useful quantitative models for predicting malignancy following an SPN observed in a routine clinic-based population.

In fact, the development of mathematical models to predict malignancy in a clinic-based population requires knowledge is some key aspects: the SPN prevalence in that setting, the variation of SPN prevalence according to relevant variables, the features of SPN in this population, and finally, the relationship between those SPN characteristics that have been associated with malignancy (such as size or morphology of the lesion) and some relevant variables. These data are relevant to estimate the clinical 'pretest' probability of malignancy of an SPN detected in a clinic-based population. Although we have data about the prevalence of SPN in screening populations [3], we are still not aware of the frequency of SPN in a routine clinic-based setting. Moreover, according to a previous systematic review [3], nodules detected in screening studies seem to be different from those detected in routine clinical practice; a fact which further justifies the need to investigate specifically this clinic-based population rather than applying the results from screening studies.

Previous lung cancer screenings evaluated the utility of this technique with or without sputum cytology [16]. However, none of these previous studies evaluated the ability of chest radiograph in the detection and characterization of the nodules. The National Lung Screening Trial [7] compares the efficacy of low-dose helical CT with chest radiograph. It shows the different prevalences of SPN and the main variables associated with them, according to the type of imaging test in a high-risk population. Nevertheless, these data are lacking in clinical practice. Although chest radiograph has a very low sensitivity for the detection and characterization of SPNs in comparison with CT, a significant number of SPNs are primarily detected with chest radiograph in clinical practice. Hence, it is essential to evaluate the different prevalence and characteristics of SPN in clinical practice for both chest radiograph and CT.

The objective of this cross-sectional study was to determine the prevalence and factors associated with SPNs, the relationship between SPN characteristics related with malignancy and relevant patient characteristics in chest radiology studies carried out in the routine practice of radiology departments for both chest radiograph and $\mathrm{CT}$, and to compare these data with the results shown in screening programs.

\section{Methods}

Patient population

A cross-sectional study of consecutive patients $\geq 35$ years referred for thoracic imaging evaluation in two hospitals in the Valencian Community (Spain) were evaluated during the years 2010 and 2011. San Juan Hospital (Alicante) and Dr. Peset Hospital (Valencia) are two tertiary centres with a catchment population of 234,424 and 377,780 people, respectively. Although both hospitals are similar, only one of the centres (Dr. Peset Hospital) included patients from the accident and emergency department. All patients referred to the radiology department from other hospital services and referrals from primary health care centres were included.

All the patients with an imaging test (chest radiograph or CT) during the period of study were included. When patients underwent both tests only the first one was recorded. Those patients who first had a chest radiograph and a CT later were categorized as having had a chest radiograph. Thus, only those patients who had a CT first were categorized as CT.

Chest radiographs were obtained with the standard technique in digital format (CR Philips at San Juan Hospital and CR Agfa at Dr. Peset Hospital). The CT technique varied according to the study that was being performed which included non-contrast $\mathrm{CT}$, contrast $\mathrm{CT}$, CT angiography and high-resolution CT. All chest CTs were obtained with a slice thickness of $1.25 \mathrm{~mm}, 120 \mathrm{KvP}$, and variable mAs according to the patient's body weight.

Patients previously diagnosed with lung cancer and patients who were not residents of the Valencian Community were excluded.

Institutional review board approval (University Miguel Hernandez Committee Ref DSP-BLL-001-10) was obtained. Given the fact that the study uses only routine data and no additional interventions, informed consent was not sought from the patients.

Data collection

Eight expert radiologists from both hospitals (four radiologists in each hospital) determined the presence of SPN in 
the thoracic study of all patients included. They used the glossary for chest radiology, which defines SPN as an opacity up to $30 \mathrm{~mm}$ in diameter, to describe SPN characteristics $[17,18]$. Thus, we limited our study to nodules between 3 and $30 \mathrm{~mm}$.

In all patients, selected variables were collected from the radiological register: type of test performed (CT or radiograph); department that ordered the test; care setting (in-patient or out-patient); reason for test (respiratory, nonrespiratory, preoperative, neoplasm, or not specified); patient characteristics (age, sex); and radiologist who performed the test.

In patients with an SPN, the radiologists described nodule characteristics in a predesigned form: a) size, expressed in millimetres and categorised as $\pm 8 \mathrm{~mm}$ in diameter, according to Fleischner criteria [17]; b) nodule shape, expressed according to the glossary previously mentioned, which considers the border as smooth or irregular (lobular or specular); c) location, upper, middle or lower lobule; and d) for those patients who underwent a $\mathrm{CT}$, nodule appearance (solid, partially solid, ground glass, or calcified). This form was completed by the radiologists simultaneously with the radiology report, in order to standardise the information.

Given the large sample of the study, we only reviewed the medical records of patients in whom an SPN was identified. The following information was collected: smoking habit (non-smokers, current, or former smokers), and previous neoplasm. Availability of information on these patients' variables (smoking habit or previous neoplasm) did not appear to be associated with the patient's other characteristics (such as age, sex, diagnostic test, reason for test, or nodule characteristics).

For the evaluation of inter-observer agreement, the first 300 tests included in the study were evaluated independently by the eight radiologists. For the intra-observer agreement, the radiologists re-evaluated 200 studies at least 6 months after the first report. In each study of observer agreement (excluding the first observation) uncertainties arising from the reports and their reasons were discussed in detail. At the end of the study on observer agreement the discrepancies and their causes were evaluated. In case of discordance, the consensus was established by the heads of the two radiology departments, the two radiologists with the most experience (I.G.A. and J.V.).

\section{Statistical analysis}

All data were computerised anonymously and sorted. Statistical precision was determined through the calculation of $95 \%$ confidence intervals using the appropriate method according to the type of measurement and the available data. All analyses were carried out with the statistical programme Stata (version 8).

We estimated the prevalence of nodules in the whole population and the prevalence according to relevant variables and their $95 \%$ CI. The effect of diverse explicative variables was considered by means of a stratified analysis and unconditional logistic regression was used (95\% confidence intervals).

To compare the SPN appearance with selected patient's characteristics, a chi squared test or Fisher's exact test was used. The variable border was regrouped into two categories according to the probability of malignancy described in previous studies [7]: smooth border or irregular border (including irregular, spiculation, and lobulation). Since there were no differences between those patients with SPN characteristics collected and those without them, the comparison only included those patients with the variables collected.

Inter- and intra-observer agreement was calculated with the kappa index, weighting with quadratic weights for ordinal categories.

\section{Results}

Inter-observer and intra-observer agreement

1) Kappa index for the detection of a SPN: a) Intra-observer agreement: kappa index ranged from 0.785 to 0.893 ; b) Inter-observer agreement: kappa index ranged from 0.793 to 0.801 (among eight radiologists);

2) Kappa index for the description of the SPN characteristics: a) Intra-observer agreement: nodule size (kappa $=0.551$ 0.526 ); nodule location (kappa $=0.565-0.759$ ); nodule edge $(\mathrm{kappa}=0.654-0.717)$; nodule appearance $(\mathrm{kappa}=0.333$ $0.793)$; and b) Inter-observer agreement: nodule size (kappa $=0.548-0.706$ ); nodule location (kappa $=0.822-$ $0.928)$; nodule edge ( $\mathrm{kappa}=0.278-0.588$ ); nodule appearance (kappa $=0.596-1.000)$.

Characteristics of patients included in the study (Table 1)

During the 2-year enrolment period, 25,529 patients with a chest image test were included in the study: 12,653 men (49.6\%) and 12,876 women (50.4\%), and the median age was 66 years (IQR 53-77; range: 35-104 years). The majority of the patients were referred from the accident and emergency department $(4,830,18.9 \%)$, surgery $(3,201,12.5 \%)$, and pulmonology $(3,051,11.9 \%)$. Most of the patients had chest radiograph $(23,102 ; 90.5 \%)$ and were out-patients $(21,613$; $84.7 \%$ ). The overall prevalence of SPN in the study was 


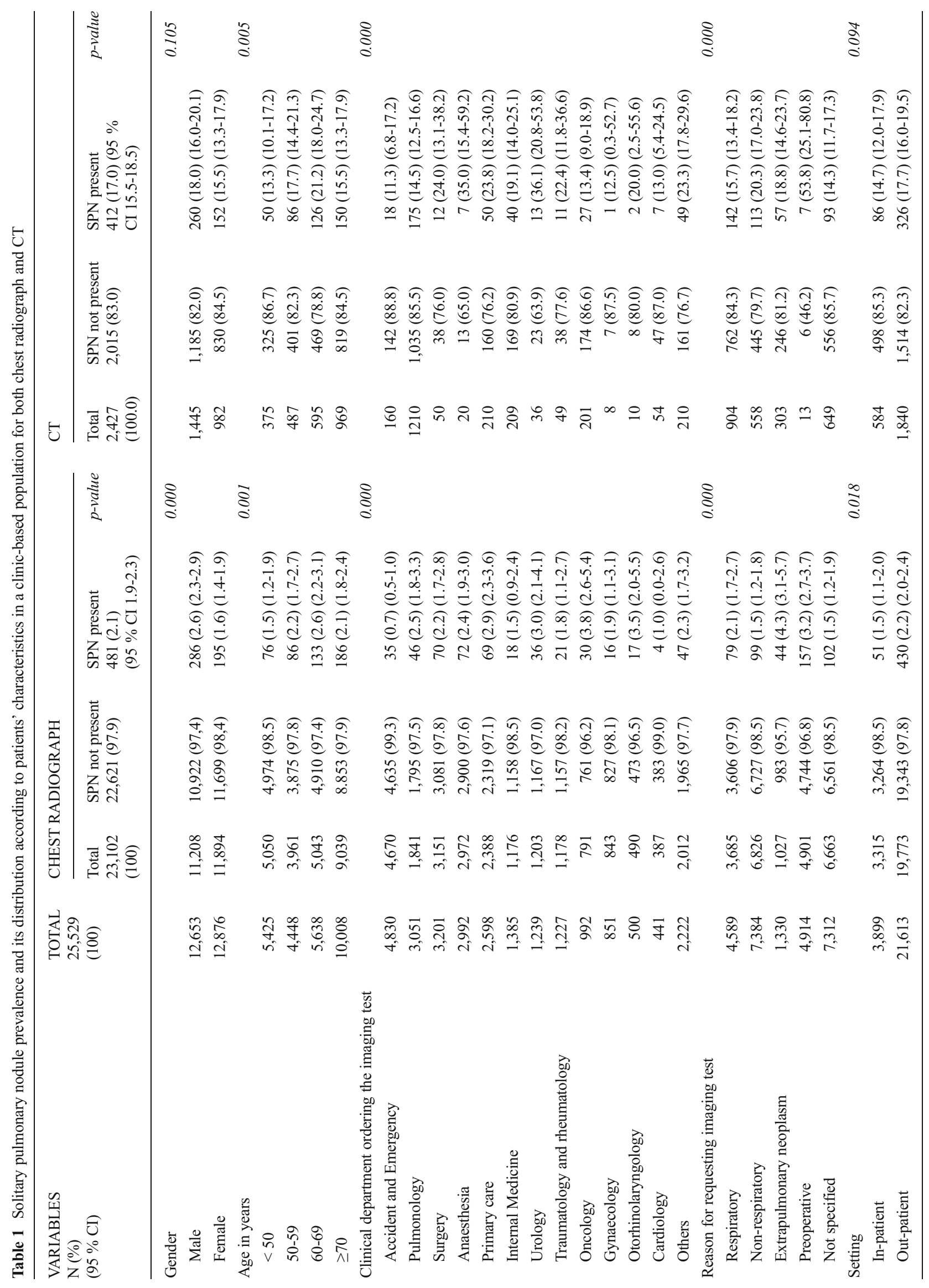


$3.5 \%$ (95\% CI $3.3-3.7)$; the prevalence was $2.1 \%$ (95\% CI $1.9-2.3)$ for chest radiograph and $17.0 \%(95 \%$ CI $15.5-$ 18.5) for CT.

Prevalence of SPN according to selected variables: univariable (Table 1) and multivariable analysis for both chest radiograph and $\mathrm{CT}$

\section{Chest radiograph}

The prevalence was $3.3 \%$ in San Juan Hospital and $1.3 \%$ in Dr. Peset Hospital $(\mathrm{p}<0.001)$. This prevalence varied according to relevant variables. Men showed a higher prevalence than women $(2.6 \%, 95 \%$ CI $2.3-3.9$ and $1.6 \%, 95 \%$ CI $1.4-1.9$, respectively, $\mathrm{p}<0.001)$. Subjects aged $60-69$ years showed the highest prevalence $(2.6 \%, 95 \%$ CI $2.2-3.1)$, while this prevalence decreased in the other age groups $(\mathrm{p}=0.001)$. The oncology department showed the greatest prevalence of SPN $(3.8 \%, 95 \%$ CI $2.6-5.4)$ in comparison with the accident and emergency department, which showed the lowest rate $(0.7 \%$, $95 \% \mathrm{CI} 0.5-1.0)$. The disorders under evaluation represented a broad range of illnesses, and the prevalence of SPN was highest in disorders categorized as extrapulmonary neoplasm $(4.3 \%, 95 \%$ CI $3.1-5.7)$ and preoperative $(3.2 \%, 95 \%$ CI 2.7 - 3.7) $(\mathrm{p}<0.001)$. The prevalence of SPN was higher in outpatients than in in-patients $(2.2 \%, 95 \%$ CI $2.0-2.4$ vs. $1.5 \%$, $95 \%$ CI $1.1-2.0, \mathrm{p}=0.018$ ).

In multivariable analysis (data not shown) adjusted by all the significant variables shown in univariable analysis, SPN were less likely to be detected in women compared to men (adjusted odds ratio $0.65 ; 95 \%$ CI $0.54-0.79, \mathrm{p}<0.001$ ). Patients older than 50 years were more likely to show an SPN when compared to patients younger than 50 years. Thus, adjusted OR for patients between $50-$ 59 years were $\mathrm{OR}=1.46$; $95 \% \mathrm{CI} 1.06-1.99 \mathrm{p}=0.016$; for those between $60-69$ years: $\mathrm{OR}=1.75 ; 95 \% \mathrm{CI}$ $1.31-2.34, \mathrm{p}<0.001$, and for those $\geq 70$ years old: $\mathrm{OR}=1.63 ; 95 \%$ CI $1.23-2.15, \mathrm{p}=0.001)$. Patients with a pre-operative request and those with a diagnosis of extrapulmonary neoplasm were more likely to show an SPN than those with an unspecified diagnosis (adjusted odds ratio 2.02; $95 \%$ CI $1.54-2.66$ and adjusted odds ratio 2.37 ; $95 \%$ CI $1.61-3.51$, respectively).

\section{$C T$}

The prevalence in San Juan Hospital was $26.5 \%$ and $12.2 \%$ in Dr. Peset Hospital. This prevalence varied according to relevant variables. Subjects aged $60-69$ showed the highest prevalence $(21.2 \%, 95 \%$ CI $18.0-24.7)$, while this prevalence decreased in the other age groups $(p=0.005)$. The urology and anaesthesia departments showed the greatest prevalence of SPN (36.1 \%, $95 \%$ CI $20.8-53.8$ and $35.0 \%, 95 \%$
CI $15.4-59.2$, respectively) in comparison with the accident and emergency department, which showed the lowest rate $(11.3 \%, 95 \%$ CI $6.8-17.2)$. The disorders under evaluation represented a broad range of illnesses, and the prevalence of SPN was highest in disorders categorized as preoperative $(53.8 \%, 95 \%$ CI $25.1-80.8)(\mathrm{p}<0.001)$. In multivariable analysis (data not shown) adjusted by all the significant variables shown in univariable analysis, patients $60-69$ years of age were more likely to show an SPN than patients younger than 50 years (adjusted odds ratio 1.78; $95 \%$ CI $1.23-2.57$, $\mathrm{p}=0.002$ ). Patients with a preoperative diagnosis and those with a diagnosis of extrapulmonary neoplasm were more likely to show an SPN than those with an unspecified diagnosis (adjusted odds ratio 7.61; $95 \%$ CI $2.41-24.04$ and adjusted odds ratio $1.60 ; 95 \%$ CI $1.05-2.43$, respectively).

Characteristics of the SPN and description of relevant variables of those patients with a SPN for both chest radiograph and CT (Table 2)

\section{Chest radiograph}

Median diameter of the SPN was $10 \mathrm{~mm}\left(\mathrm{P}_{25}-\mathrm{P}_{75}: 7-14 \mathrm{~mm}\right)$. More than half of the nodules were larger than $8 \mathrm{~mm}(59.7 \%$, $95 \%$ CI $55.1-64.1)$. SPN was more frequently located in the upper lobe (54.5\%, $95 \%$ CI $50.0-60.0)$. An irregular or not well defined border was found in 144 nodules $(29.9 \%$, $95 \%$ CI 25.9 - 34.2) and the spiculated border was described in 62 nodules. A high percentage of patients with an SPN did not have a previous malignancy $(65.9 \%, 95 \%$ CI $61.5-70.1)$ and were former or current smokers $(59.0 \%, 95 \%$ CI $54.5-63.5)$.

\section{$C T$}

Median diameter of the SPN was $8 \mathrm{~mm}\left(\mathrm{P}_{25} \mathrm{P}_{75}: 5-13 \mathrm{~mm}\right)$. Half of the nodules were smaller than $8 \mathrm{~mm}(51.3 \%, 95 \% \mathrm{CI}$ 46.4 - 56.2). SPN was more frequently located in the upper lobe $(48.1 \%, 95 \%$ CI 43.1 - 53.0). An irregular or not well-defined border was found in 151 nodules $(36.6 \%, 95 \%$ CI $32.0-41.5$ ) and the spiculated border was described in 44 nodules. More than half of the nodules had a solid appearance $(225 ; 54.6 \%, 95 \%$ CI $49.8-59.4)$, the frequency of groundglass opacity was $6.1 \%$, (95\% CI $4.0-8.8)$, and the percentage of partly solid nodules was $3.4 \%$ (95\% CI 1.9 5.6). Nearly $70 \%$ of patients with an SPN did not have a previous malignancy $(69.9 \%, 95 \%$ CI $65.2-74.3)$, and $60.4 \%$, $(95 \%$ CI $55.5-65.2)$ were former or current smokers. 
Table 2 Characteristics of the 893 SPN detected in the imaging tests carried out in the study, and the description of relevant variables of patients with an SPN for both chest radiograph and $\mathrm{CT}$

\begin{tabular}{|c|c|c|c|c|c|c|}
\hline \multirow[t]{2}{*}{ CHARACTERISTICS } & \multicolumn{3}{|c|}{ CHEST RADIOGRAPH } & \multicolumn{3}{|l|}{ CT } \\
\hline & $\mathrm{N}$ & $\%$ & $95 \% \mathrm{CI}$ & $\mathrm{N}$ & $\%$ & $95 \% \mathrm{CI}$ \\
\hline \multicolumn{7}{|l|}{ NODULE } \\
\hline \multicolumn{7}{|l|}{ Diameter (mm) } \\
\hline$<8$ & 168 & 34.9 & $(30.7-39.4)$ & 212 & 51.3 & $(46.4-56.2)$ \\
\hline$>8$ & 287 & 59.7 & $(55.1-64.1)$ & 188 & 45.5 & $(40.6-50.5)$ \\
\hline Not specified & 25 & 5.2 & $(3.4-7.6)$ & 13 & 3.1 & $(1.7-5.3)$ \\
\hline \multicolumn{7}{|l|}{ Localization } \\
\hline Upper lobe & 262 & 54.5 & $(50.0-60.0)$ & 198 & 48.1 & $(43.1-53.0)$ \\
\hline Lower lobe & 39 & 8.1 & $(5.8-10.9)$ & 50 & 12.1 & $(9.1-15.7)$ \\
\hline Middle lobe & 160 & 33.3 & $(29.1-37.7)$ & 150 & 36.4 & $(31.8-41.3)$ \\
\hline Not specified & 20 & 4.2 & $(2.6-6.3)$ & 14 & 3.4 & $(1.9-5.6)$ \\
\hline \multicolumn{7}{|l|}{ Border } \\
\hline Smooth border or well defined border & 128 & 26.6 & $(22.7-30.8)$ & 88 & 21.4 & $(17.5-25.6)$ \\
\hline Irregular or not well defined & 144 & 29.9 & $(25.9-34.2)$ & 151 & 36.6 & $(32.0-41.5)$ \\
\hline - Spiculation & 62 & 43.1 & $(34.8-51.6)$ & 44 & 29.1 & $(22.0-37.1)$ \\
\hline - Lobulation & 33 & 22.9 & $(16.3-30.7)$ & 41 & 27.2 & $(20.2-35.0)$ \\
\hline - Other & 49 & 34.0 & $(26.3-42.4)$ & 66 & 43.7 & $(35.7-52.0)$ \\
\hline Not specified & 209 & 43.5 & $(39.0-48.0)$ & 173 & 42.0 & $(37.2-46.9)$ \\
\hline \multicolumn{7}{|l|}{ Opacity on CT } \\
\hline Solid & & & & 225 & 54.6 & $(49.7-59.5)$ \\
\hline Ground glass & & & & 25 & 6.1 & $(4.0-8.8)$ \\
\hline Calcification & & & & 16 & 3.9 & $(2.2-6.2)$ \\
\hline Partly solid & & & & 14 & 3.4 & $(1.9-5.6)$ \\
\hline Not specified & & & & 132 & 32.0 & $(27.6-36.8)$ \\
\hline \multicolumn{7}{|l|}{ PATIENT } \\
\hline \multicolumn{7}{|l|}{ Previous malignancy } \\
\hline No & 317 & 65.9 & $(61.5-70.1)$ & 288 & 69.9 & $(65.2-74.3)$ \\
\hline Yes & 164 & 34.1 & $(29.9-38.5)$ & 124 & 30.1 & $(25.7-34.8)$ \\
\hline \multicolumn{7}{|l|}{ Smoking habit } \\
\hline No & 100 & 20.8 & $(17.2-24.7)$ & 86 & 20.9 & $(17.0-25.1)$ \\
\hline Former and current & 284 & 59.0 & $(54.5-63.5)$ & 249 & 60.4 & $(55.5-65.2)$ \\
\hline Not specified & 97 & 20.2 & $(16.7-24.0)$ & 77 & 18.7 & $(15.0-22.8)$ \\
\hline Total & 481 & 100.0 & & 412 & 100.0 & \\
\hline
\end{tabular}

Characteristics of SPN according to selected variables

We also tested the difference in SPN size (diameter), border (regular or irregular), and location (upper, middle, or lower) according to selected patient's characteristics for both chest radiograph and $\mathrm{CT}$. We only included patients with nodule characteristics available (data not shown). We describe the significant associations:

\section{Chest radiograph}

- Border: Smokers were more likely to show nodules with irregular border than non-smokers $(96 / 166 ; 57.8 \%$ vs. $23 / 55 ; 41.8 \%$; $=0.039$ )
$C T$

- Size:

- $\quad$ Patients older than 70 years $(77 / 145 ; 53.1 \%)$ and those aged $60-69$ years $(60 / 121 ; 49.6 \%)$ were more likely to show a nodule larger than $8 \mathrm{~mm}$ than patients aged 50 59 years $(33 / 83 ; 39.8 \%)$ and those younger than 50 years $(15 / 49 ; 30.6 \%)(\mathrm{p}=0.023)$.

- Men were more likely to show a nodule larger than $8 \mathrm{~mm}$ than women $(126 / 250 ; 50.4 \%$ vs. 59/148; $39.9 \%$; 0.042)

- $\quad$ Patients with a respiratory diagnosis $(67 / 137 ; 48.9 \%)$ were more likely to show a nodule larger than $8 \mathrm{~mm}$ than patients with other diagnoses such as extrapulmonary neoplasm $(17 / 55 ; 30.9 \%)(\mathrm{p}=0.046)$. 
- Border: Men were more likely to show a nodule with irregular border than women $(106 / 156 ; 67.9 \%$ vs. 45/83; $54.2 \% ; \mathrm{p}=0.036$ )

\section{Discussion}

The study shows an important prevalence of newly diagnosed SPN $2.1 \%$ (95 \% CI $1.9-2.3$ ) for chest radiograph and $17.0 \%$ (95\% CI 15.5 - 18.5) for CT in the imaging tests of the 25,529 patients included in the study. For both types of imaging tests, this prevalence varied according to age, reason for test, and clinical department. Moreover, in patients undergoing a chest radiograph, the prevalence varied according to sex and setting. Patients older than 50 years or those who were undergoing chest imaging in the course of a preoperative examination for extrapulmonary neoplasm were more likely to show an SPN. Men and outpatients having a chest radiograph were also more likely to show an SPN. In relation to SPN characteristics, nodules detected by radiograph were larger than $8 \mathrm{~mm}$, as opposed to nodules detected by CT. However, for both types of tests, detected nodules were mainly located in the upper lobes and with irregular borders. In those patients who had an SPN detected by chest radiograph, irregular border was associated with smoking (current and former). In patients who had a CT, a size exceeding $8 \mathrm{~mm}$ was associated with smoking. SPNs appeared to be associated with age $\geq 60$ years, diagnosis of a respiratory illness, or male gender. In addition, an irregular border was also associated with sex (men).

Recent screening studies in high-risk populations using low-dose CT reported a higher SPN prevalence than in our study $(73.7 \%$ in the PanCan Study [9] and $27.9 \%$ in the National Lung Screening Trial [8], vs. $17.0 \%$ in our study). However, according to previous screening studies using CT, the prevalence of SPNs ranged from 8 to $51 \%$ [3]. The SPN prevalence in the screening studies using radiograph was also higher than that of our study $(6.2 \%$ in the National Lung Screening Trial [8] vs. $2.1 \%$ in our study). Previous hospitalbased studies using chest radiograph to detect SPN also showed a higher prevalence (23 to $75 \%$ ); however, as we previously mentioned, the lack of description of patients' features or the highly selected population could have affected the results of these studies $[6,13]$. The lower SPN prevalence shown in our study could be explained by the unselected nature of our clinical population by including patients having chest imaging from all clinical departments including the accident and emergency department.

For both, patients having a chest radiograph and those having a CT, the prevalence of SPNs was higher in those patients with a diagnosis of neoplasm ( $4.3 \%$ for chest radiograph and $18.8 \%$ for CT) or those undergoing a preoperative examination (3.2\% for chest radiograph and $53.8 \%$ for CT) compared to patients with other diagnoses. According to a previous study [19], $75 \%$ of patients with extrapulmonary carcinoma or sarcoma who had a CT showed a SPN, whereas our study showed a lower percentage, probably due to the inclusion of an unselected population in our study.

Most of the detected SPNs had characteristics that have been associated with malignancy according to previous quantitative models [7]. A systematic literature review carried out in 2007 by the ACCP [3] described that irregular, spiculated, and lobulated nodule borders were more predictive of malignancy than smooth edges. Nodule size (SPN bigger than $8 \mathrm{~mm}$, according to Fleischner recommendations [17],) and nodule location (those SPN located in upper lobe are more likely to be malignant [20]) are also related with malignancy. In our study, for patients who had both a chest radiograph and a CT, and taking into account only those patients with a nodule characterization, less than half of them showed smooth edges. Of the SPN detected by chest radiology, $59.7 \%$ were larger than $8 \mathrm{~mm}$. This percentage is lower than the data shown in the National Lung Screening Study, where more than $70 \%$ of the SPN detected by chest radiograph were larger than $8 \mathrm{~mm}$. Regarding SPN observed by CT, $44.9 \%$ were larger than $8 \mathrm{~mm}$, which is similar to the $43 \%$ found in the National Lung Screening Study. Thus, according to nodule size, the pretest probability of malignancy could be lower in our study than in screening studies.

Both ground-glass and partially solid nodules are considered subsolid nodules in contrast with solid nodules [17]. Regarding the malignancy of nodules according to the different types of opacity, different opinions exist in the literature; previously some studies [21] found ground-glass opacities to be associated with malignancy, but other studies did not include these characteristics in their prediction models [9]. However, when partially solid and groundglass nodules were pooled [22], the prevalence of malignancy was higher than for solid nodules. In any case, according to Fleischner recommendations [17], these types of nodules should be managed conservatively. In our study, the percentage of ground-glass nodules was $6.1 \%$ and $3.4 \%$ for partially solid nodules. Although the frequency of ground-glass nodules is lower than those shown, for example, in the Pan Can Study [9] (15.8\%), the frequency of subsolid nodules is higher than those shown in other screening studies, such as the Nelson study [23]. Thus, the likelihood of malignancy in our population could be high.

Although the prevalence of SPN observed in our study in much lower than in screening studies, the proportion of cases with irregular nodules (both for patients with a chest radiograph and those with a CT), is similar to that observed in screening studies [3]. In our study, taking into account only those patients with a nodule characterization, $36.8 \%$ of the patients with a CT showed smooth nodules, in comparison with previous studies using $\mathrm{CT}$, where the prevalence of 
smooth nodules varied from 20 to $44 \%$. However, we were unable to obtain data related to SPN morphology in some cases, making it difficult to compare the results.

Some patient characteristics such as sex, advanced age, or history of smoking have been associated with a higher risk of malignant SPN [24]. In our study, men, advanced age, respiratory disease, and current or former smoking were associated with SPN characteristics related to malignancy, such as nodule diameter or irregular border. In our study, in patients who had a chest radiograph, smokers were more likely to show nodules with irregular edges than non-smokers. Swensen [4] reported an increased likelihood of malignancy with the higher number of cigarettes per day. Although we did not quantify the smoking habit, it seems to be associated with SPN characteristics related with malignancy, as would be expected. In patients having a $\mathrm{CT}$, men were more likely to show nodules bigger than $8 \mathrm{~mm}$ and nodules with irregular edges than women. Moreover, patients $\geq 60$ years old were more likely to show nodules bigger than $8 \mathrm{~mm}$. According to previous studies [7, 25], the patient's age is one of the most important clinical factors associated with malignancy. In fact, Swensen [4] described a 2.1 likelihood ratio of malignancy in patients 60 to 69 years of age, and a 5.7 in patients 70 to 83 years of age. In multivariable analysis, the patient's previous malignancy was not associated with any SPN variables related with malignancy. In previous studies, the relationship between lung cancer and previous malignancy is not clear [6]. However, some morphologic variables such as spiculation or irregular borders may dilute the association in multivariable analysis given that metastases are usually well defined and have no spiculation.

This study presents new data about SPN detected in a clinic-based population for both chest radiograph and CT, but some limitations should be addressed. The observer variability in the determination of the presence of nodules and their characteristics was less than perfect and should be acknowledged as one limitation of the study. This limitation in imaging studies has been shown in other studies [26]. All the radiologists who participated in the study followed the same criteria for the detection and characterization of nodules. Moreover, the evaluation of observer agreement, its causes and the determination of a consensus with the participation of two experts helped to limit the impact of this problem in the overall study findings.

The lack of electronic medical records meant that we were unable to retrieve complete information for a relatively high proportion of cases. Incomplete information on some patients' data, such as smoking habit, occupational exposure, or family history, could lead to information bias. However, there were no significant differences with respect to other patient's characteristics (such as age, sex, diagnostic test, reason for test, or nodule characteristics) between patients with the available data and those without.
Despite the similar populations, and the use by radiologists of similar protocols to detect SPN, we observed a different SPN prevalence between the two hospitals included in the study. The reason for this difference could be due to several factors; in addition to the usual inter-reader variation observed when ordering a diagnostic test [27], there were some differences related to the type of clinical departments included in each hospital. Only the hospital that showed the lowest prevalence of SPN included the 4,830 imaging tests carried out in the emergency department (the prevalence of SPN in this department is $1.1 \%$ ). Moreover, $86 \%$ of the imaging tests ordered from primary care were included in the hospital that showed the highest prevalence (the prevalence of SPN in the primary care department was $4.6 \%$ ).

As opposed to previous studies [28], we did not exclude patients with a malignancy outside the chest because we wanted to show the whole spectrum of patients with an SPN in a clinic-based population. This study differs in many ways from previous evaluations of SPNs. Our study is based on information from consecutive radiology reports, representative of the general clinic-based population in two health districts and it is not biased by patient selection. It should be underlined that in order to establish the predictive value of these, SPN and patient characteristics for lung cancer will be ascertained once follow-up of the cohort, now under investigation, will be accomplished.

In conclusion, we have shown that SPNs may be observed in one of every six patients undergoing $\mathrm{CT}$ and in one of every 47 patients undergoing a chest radiograph in a routine clinical setting. This was lower than that shown in screening studies using a highly selected population. Some significant differences related to SPN characteristics have also been shown between our population and those included in screening studies, such as nodule size (smaller in our study than in screening studies). Moreover, some patient characteristics such as age, sex, respiratory disease, or smoking habit were associated with nodule characteristics that are known to be associated to malignancy (bigger nodule size or irregular border).

Given the difference in nodule characteristics and variables associated with the nodule prevalence for patients who have had a CT or a chest radiograph, it is important to study these techniques separately in the general population. Once longterm risk of cancer is determined, these results will be applicable to estimate the clinical 'pretest' probability of malignancy of a SPN detected in a clinic-based population for both chest radiograph and $\mathrm{CT}$.

Acknowledgments The scientific guarantor of this publication is Prof. Ildefonso Hernandez Aguado, Head of the Department of Public Health, Gynecology and History of Medicine, Miguel Hernandez University. The authors of this manuscript declare no relationships with any companies, whose products or services may be related to the subject matter of the article. This study has received funding by Instituto de Salud Carlos III (Minister of Science, Spain) (Ref. PI09/0477) and partial funding and 
support by the CIBER en Epidemiología y Salud Pública (CIBERESP) in Spain. No complex statistical methods were necessary for this paper. Institutional Review Board approval was obtained: The ethical committee of the Miguel Hernandez University approved the study protocol (ref DSP-BLL-001-10). Written informed consent was not required for this study because only secondary data were included. Methodology: Cross sectional study / multicentre study.

Open Access This article is distributed under the terms of the Creative Commons Attribution Noncommercial License which permits any noncommercial use, distribution, and reproduction in any medium, provided the original author(s) and the source are credited.

\section{References}

1. Postma TJBM, Alers JC, Terpstra S, Zuurbier A (2006) The future of imaging techniques for cancer patients in The Netherlands: A Delphi study. Eur J Health Econ 7:117-122

2. Thrall JH (1994) The radiologist in the 1990s: new practice expectations and management responsibilities. AJR Am J Roentgenol 163: $11-15$

3. Wahidi MM, Govert JA, Goudar RK, Gould MK, McCrory DC, American College of Chest Physicians (2007) Evidence for the treatment of patients with pulmonary nodules: when is it lung cancer? An ACCP evidence-based clinical practice guideline (2nd edition). Chest 132:94S-107S

4. Swensen SJ, Silverstein MD, Edell ES et al (1999) Solitary pulmonary nodules: clinical prediction model versus physicians. Mayo Clin Proc 74:319-329

5. Gould MK, Donington J, Lynch WR et al (2013) Evaluation of individuals with pulmonary nodules: when is it lung cancer? Diagnosis and management of lung cancer, 3rd ed: American College of Chest Physicians evidence-based clinical practice guidelines. Chest 143:e93S-e120S

6. Gould MK, Fletcher J, Iannettoni MD, American College of Chest Physicians et al (2007) Evaluation of patients with pulmonary nodules: when is it lung cancer?: ACCP evidence-based clinical practice guidelines (2nd edition). Chest 132:108S-130S

7. Aberle DR, DeMello S, Berg CD et al (2013) National Lung Screening Trial Research Team. Results of the two incidence screenings in the National Lung Screening Trial. N Engl J Med 369:920-931

8. Tammemägi MC, Katki HA, Hocking WG et al (2013) Selection criteria for lung-cancer screening. N Engl J Med 368:728-736

9. McWilliams A, Tammemagi MC, Mayo JR et al (2013) Probability of cancer in pulmonary nodules detected on first screening CT. N Engl J Med 369:910-919

10. Zerhouni EA, Stitik FP, Siegelman SS et al (1986) CT of the pulmonary nodule: a cooperative study. Radiology 160:319-327

11. Siegelman SS, Khouri NF, Leo FP et al (1986) Solitary pulmonary nodules: CT assessment. Radiology 160:307-312

12. Tozaki M, Ichiba N, Fukuda K (2005) Dynamic magnetic resonance imaging of solitary pulmonary nodules: utility of kinetic patterns in differential diagnosis. J Comput Assist Tomogr 29:13-19
13. Swensen SJ, Silverstein MD, Ilstrup DM et al (1997) The probability of malignancy in solitary pulmonary nodules: application to small radiologically indeterminate nodules. Arch Intern Med 157:849-855

14. Matsuki Y, Nakamura K, Watanabe H et al (2002) Usefulness of an artificial neural network for differentiating benign from malignant pulmonary nodules on high-resolution CT: evaluation with receiver operating characteristic analysis. AJR Am J Roentgenol 178:657-663

15. Marcus PM, Bergstralh EJ, Fagerstrom RM, Williams DE, Fontana R, Taylor WF, Prorok PC (2000) Lung cancer mortality in the Mayo Lung Project: impact of extended follow-up. J Natl Cancer Inst 92: 1308-1316

16. Melamed MR (2000) Lung cancer screening results in the National Cancer Institute New York study. Cancer 89(11 Suppl):2356-2362

17. Bankier AA, MacMahon H, McLoud TC, Müller NL, Remy J (2008) Fleischner Society: glossary of terms for thoracic imaging. Radiology 246:697-722

18. MacMahon H (2005) Guidelines for management of small pulmonary nodules detected on CT scans: a statement from the Fleischner Society. Radiology 237:395-400

19. Hanamiya M, Aoki T, Yamashita Y, Kawanami S, Korogi Y (2012) Frequency and significance of pulmonary nodules on thin-section CT in patients with extrapulmonary malignant neoplasms. Eur J Radiol $81: 152-157$

20. Sone S, Li F, Yang ZG, Takashima S et al (2000) Characteristics of small lung cancers invisible on conventional chest radiograph and detected by population based screening using spiral CT. Br J Radiol 73:137-145

21. Patel VK, Naik SK, Naidich DP et al (2013) A practical algorithmic approach to the diagnosis and management of solitary pulmonary nodules: part 1: radiologic characteristics and imaging modalities. Chest 143:825-839

22. Li Y, Chen KZ, Wang J (2011) Development and validation of a clinical prediction model to estimate the probability of malignancy in solitary pulmonary nodules in Chinese people. Clin Lung Cancer 12: 313-319

23. Li F, Sone S, Abe H, Macmahon H, Doi K (2004) Malignant versus benign nodules at $\mathrm{CT}$ screening for lung cancer: comparison of thinsection CT findings. Radiology 233:793-798

24. Asamura H, Suzuki K, Watanabe S, Matsuno Y, Maeshima A, Tsuchiya R (2003) A clinicopathological study of resected subcentimeter lung cancers: a favorable prognosis for ground glass opacity lesions. Ann Thorac Surg 76:1016-1022

25. Xu DM, van der Zaag-Loonen HJ, Oudkerk M et al (2009) Smooth or attached solid indeterminate nodules detected at baseline CT screening in the NELSON study: cancer risk during 1 year of follow-up. Radiology 250:264-272

26. Pinsky PF, Gierada DS, Nath PH, Kazerooni E, Amorosa J, National Lung Screening Trial (2013) Variability in Nodule Detection Rates in Chest CT Studies. Radiology 268:865-873

27. Verstappen WH, ter Riet G, Dubois WI, Winkens R, Grol RP, van der Weijden T (2004) Variation in test ordering behaviour of GPs: professional or context-related factors? Fam Pract 21:387-395

28. Alzahouri K, Velten M, Arveux P, Woronoff-Lemsi MC, Jolly D, Guillemin F (2008) Management of SPN in France. Pathways for definitive diagnosis of solitary pulmonary nodule: a multicentre study in 18 French districts. BMC Cancer 8:93 